\title{
On Generalized Fractional Integral Operators and the Generalized Gauss Hypergeometric Functions
}

\author{
Dumitru Baleanu ${ }^{1,2,3}$ and Praveen Agarwal ${ }^{4}$ \\ ${ }^{1}$ Department of Chemical and Materials Engineering, Faculty of Engineering, King Abdulaziz University, \\ P.O. Box 80204, Jeddah 21589, Saudi Arabia \\ ${ }^{2}$ Department of Mathematics and Computer Sciences, Faculty of Art and Sciences, Cankaya University, Ankara, Turkey \\ ${ }^{3}$ Institute of Space Sciences, P.O. Box MG-23, Magurele, 76900 Bucharest, Romania \\ ${ }^{4}$ Department of Mathematics, Anand International College of Engineering, Jaipur 303012, India
}

Correspondence should be addressed to Dumitru Baleanu; dumitru.baleanu@gmail.com

Received 3 February 2014; Accepted 19 March 2014; Published 22 April 2014

Academic Editor: Ivanka Stamova

Copyright (C) 2014 D. Baleanu and P. Agarwal. This is an open access article distributed under the Creative Commons Attribution License, which permits unrestricted use, distribution, and reproduction in any medium, provided the original work is properly cited.

A remarkably large number of fractional integral formulas involving the number of special functions, have been investigated by many authors. Very recently, Agarwal (National Academy Science Letters) gave some integral transform and fractional integral formulas involving the $F_{p}^{(\alpha, \beta)}(\cdot)$. In this sequel, here, we aim to establish some image formulas by applying generalized operators of the fractional integration involving Appell's function $F_{3}(\cdot)$ due to Marichev-Saigo-Maeda. Some interesting special cases of our main results are also considered.

\section{Introduction}

In recent decades, several extensions of the well-known special functions like beta function, gamma functions, and Gauss hypergeometric functions and their properties started to be one of the main directions for several researchers (see, e.g., $[1-4])$.

In the sequel, recently, Özergin et al. introduced and studied some fundamental properties and characteristics of the generalized Gauss hypergeometric functions $F_{p}^{(\alpha, \beta)}(\cdot)$ which are defined by (see, e.g., [4, page 4606, Section 3]; see also [3, page 39, Chapter 4])

$$
\begin{array}{r}
F_{p}^{(\alpha, \beta)}(a, b ; c ; z):=\sum_{n=0}^{\infty}(a)_{n} \frac{B_{p}^{(\alpha, \beta)}(b+n, c-b)}{B(b, c-b)} \frac{z^{n}}{n !} \\
(|z|<1),
\end{array}
$$

$(\min (\mathfrak{R}(\alpha), \mathfrak{R}(\beta))>0 ; \mathfrak{R}(c)>\Re(b)>0$ and $\mathfrak{R}(p) \geq 0)$, where $B_{p}^{(\alpha, \beta)}(\cdot, \cdot)$ is the generalized beta type function defined by (see, e.g., [4, page 4602, Equation (4)]; see also [3, page 32, Chapter 4])

$$
B_{p}^{(\alpha, \beta)}(x, y)=\int_{0}^{1} t^{x-1}(1-t)^{y-1} \cdot{ }_{1} F_{1}\left(\alpha ; \beta ; \frac{-p}{t(1-t)}\right) d t,
$$

$\left(\mathfrak{R}(p)>0 ;(\Re(x), \mathfrak{R}(y), \mathfrak{R}(\alpha), \mathfrak{R}(\beta))>0\right.$ and $B_{0}^{(\alpha, \beta)}(x, y)=$ $B(x, y))$, and $B(x, y)$ is the well-known Euler's beta function defined by

$$
B(x, y):=\int_{0}^{1} t^{x-1}(1-t)^{y-1} d t \quad(\Re(x)>0, \Re(y)>0) .
$$

Indeed, in their special case when $p=0$, the function $F_{p}^{(\alpha, \beta)}(\cdot)$ would reduce immediately to the extensively investigated Gauss hypergeometric function ${ }_{2} F_{1}(\cdot)$. The ${ }_{2} F_{1}(\cdot)$ is 
the special case of the well-known generalized hypergeometric series ${ }_{p} F_{q}(\cdot)$ defined by (see, e.g., [5, Section 1.5$]$ )

$$
\begin{aligned}
{ }_{p} F_{q}\left[\begin{array}{c}
\alpha_{1}, \ldots, \alpha_{p} ; \\
\beta_{1}, \ldots, \beta_{q} ;
\end{array}\right] & z=\sum_{n=0}^{\infty} \frac{\left(\alpha_{1}\right)_{n} \cdots\left(\alpha_{p}\right)_{n}}{\left(\beta_{1}\right)_{n} \cdots\left(\beta_{q}\right)_{n}} \frac{z^{n}}{n !} \\
& ={ }_{p} F_{q}\left(\alpha_{1}, \ldots, \alpha_{p} ; \beta_{1}, \ldots, \beta_{q} ; z\right),
\end{aligned}
$$

where $(\lambda)_{n}$ is the Pochhammer symbol defined (for $\lambda \in \mathbb{C}$ ) by (see [5, page 2 and pages $4-6]$ )

$$
\begin{aligned}
(\lambda)_{n} & :=\frac{\Gamma(\lambda+n)}{\Gamma(\lambda)} \\
& = \begin{cases}1 & (n=0 ; \lambda \in \mathbb{C} \backslash 0) \\
\lambda(\lambda+1) \cdots(\lambda+n-1) & (n \in \mathbb{N} ; \lambda \in \mathbb{C}) .\end{cases}
\end{aligned}
$$

It is being assumed conventionally that $(0)_{0}:=1$ and understood tacitly that the $\Gamma$-quotient exists.

The above-mentioned detailed and systematic investigation by Özergin [3] was indeed motivated largely by the demonstrated potential for applications of the generalized Gauss hypergeometric function $F_{p}^{(\alpha, \beta)}(\cdot)$ and their special cases in many diverse areas of mathematical, physical, engineering, and statistical sciences (see $[3,4]$ and the references cited therein).

Fractional integral operators are widely used to solve differential equations and integral equations. So a lot of work has been done on the theory and applications of fractional integral operators. Most popular fractional integral transforms are due to Saigo, Erdélyi, Kober, Riemann, Liouville, Weyl, and so on (see, e.g., [6-16]). Here, in this paper, we use the Marichev-Saigo-Maeda type fractional integral operator to derive certain image formulas for the generalized Gauss hypergeometric function $F_{p}^{(\alpha, \beta)}(\cdot)$. We also consider some interesting special cases and consequences of our main results.

\section{Generalized Fractional Integration Operators and Their Applications}

This section deals with the composition formulae for the generalized Gauss hypergeometric type functions $F_{p}^{(\alpha, \beta)}(\cdot)$. The results are given in the form of theorems. For the purpose of these results, we use the following fractional integral operators due to Marichev-Saigo-Maeda.

For $x>0$ and $\mu, \mu^{\prime}, \nu, \nu^{\prime}, \eta \in \mathbb{C}(\mathfrak{R}(\eta)>0)$, we have

$$
\begin{aligned}
& \left(I_{0, x}^{\mu, \mu^{\prime}, v, \nu^{\prime}, \eta} f\right)(x) \\
& :=\frac{x^{-\mu}}{\Gamma(\eta)} \int_{0}^{x}(x-t)^{\eta-1} t^{-\mu^{\prime}} F_{3} \\
& \quad \times\left(\mu, \mu^{\prime}, \nu, v^{\prime} ; \eta ; 1-\frac{t}{x}, 1-\frac{x}{t}\right) f(t) d t
\end{aligned}
$$

$$
\begin{aligned}
& \left(I_{x, \infty}^{\mu, \mu^{\prime}, v, \nu^{\prime}, \eta} f\right)(x) \\
& \quad:=\frac{x^{-\mu^{\prime}}}{\Gamma(\eta)} \int_{x}^{\infty}(t-x)^{\eta-1} t^{-\mu} F_{3} \\
& \quad \times\left(\mu, \mu^{\prime}, \nu, \nu^{\prime} ; \eta ; 1-\frac{x}{t}, 1-\frac{t}{x}\right) f(t) d t,
\end{aligned}
$$

where the function $f(t)$ is so constrained that the defining integrals in (6) exist.

These operators (integral transforms) were introduced by Marichev [12] as Mellin type convolution operators with the Appell function $F_{3}(\cdot)$ in their kernel. These operators were rediscovered and studied by Saigo in [14] as generalization of the so-called Saigo fractional integral operators; see [10]. The properties of these operators were studied by Saigo and Maeda [15], in particular, relations of operators with the Mellin transforms, hypergeometric operators (or Saigo fractional integral operators), their decompositions, and acting properties in the McBride spaces $F_{p ; \mu}$ (see [13]).

In (6), the symbol $F_{3}(\cdot)$ denotes the so-called 3rd Appell function (known also as Horn function) (see [17, page 413]):

$$
\begin{array}{r}
F_{3}\left(\mu, \mu^{\prime}, \nu, \nu^{\prime} ; \eta ; x ; y\right)=\sum_{m, n=0}^{\infty} \frac{(\mu)_{m}\left(\mu^{\prime}\right)_{n}(\nu)_{m}\left(\nu^{\prime}\right)_{n}}{(\eta)_{m+n}} \frac{x^{m}}{m !} \frac{y^{n}}{n !} \\
(\max \{|x|,|y|\}<1) .
\end{array}
$$

The properties of this function and some reduction formulas are discussed in [17, page 412-415] and [16], respectively.

In the sequel, following Saigo and Maeda [15], the lefthand side and right-hand side generalized integrations of the type (6) for a power function are given by

$$
\begin{aligned}
& \left(I_{0, x}^{\mu, \mu^{\prime}, \nu, \nu^{\prime}, \eta} t^{\rho-1}\right)(x) \\
& =\frac{\Gamma(\rho) \Gamma\left(\rho+\eta-\mu-\mu^{\prime}-\nu\right) \Gamma\left(\rho+\nu^{\prime}-\mu^{\prime}\right)}{\Gamma\left(\rho+\nu^{\prime}\right) \Gamma\left(\rho+\eta-\mu-\mu^{\prime}\right) \Gamma\left(\rho+\eta-\mu^{\prime}-v\right)} \\
& \quad \times x^{\rho+\eta-\mu-\mu^{\prime}-1},
\end{aligned}
$$

$\left(\mathfrak{R}(\eta)>0 ; \mathfrak{R}(\rho)>\max \left\{0, \mathfrak{R}\left(\mu+\mu^{\prime}+\nu-\eta\right), \mathfrak{R}\left(\mu^{\prime}-\nu^{\prime}\right)\right\}\right)$ and

$$
\begin{aligned}
\left(I_{x, \infty}^{\mu, \mu^{\prime}, \nu, \nu^{\prime}, \eta} t^{\rho-1}\right)(x) \\
=\frac{\Gamma(1-\rho-\nu) \Gamma\left(1-\rho-\eta+\mu+\mu^{\prime}\right) \Gamma\left(1-\rho+\mu+\nu^{\prime}-\eta\right)}{\Gamma(1-\rho) \Gamma\left(1-\rho+\mu+\mu^{\prime}+\nu^{\prime}-\eta\right) \Gamma(1-\rho+\mu-\nu)} \\
\quad \times x^{\rho+\eta-\mu-\mu l-1},
\end{aligned}
$$

$\left(\mathfrak{R}(\eta)>0 ; 0<\mathfrak{R}(\rho)<1+\min \left\{\mathfrak{R}(-\nu), \mathfrak{R}\left(\mu+\mu^{\prime}-\eta\right), \mathfrak{R}(\mu+\right.\right.$ $\left.\left.\left.\nu^{\prime}-\eta\right)\right\}\right)$.

We now establish image formulas for the generalized Gauss hypergeometric function involving Saigo-Maeda fractional integral operators (6), which are expressed in terms of 
the generalized Gauss hypergeometric type function ${ }_{3} F_{p, 3}^{(\alpha, \beta)}$. These formulas are given in Theorems 1 and 2 below.

For convenience, we defined the generalized Gauss hypergeometric type function ${ }_{3} F_{p, 3}^{(\alpha, \beta)}$ as follows:

$$
\begin{aligned}
{ }_{3} F_{p, 3}^{(\alpha, \beta)} & {\left[\begin{array}{c}
a, b, d, e, f \\
c, g, h, k
\end{array} ; z\right] } \\
:= & \sum_{n=0}^{\infty}(a)_{n} \\
& \times \frac{(d)_{n}(e)_{n}(f)_{n} B_{p}^{(\alpha, \beta)}(b+n, c-b)}{(g)_{n}(h)_{n}(k)_{n} B(b, c-b)} \frac{z^{n}}{n !} \quad(|z|<1),
\end{aligned}
$$

$(\min (\mathfrak{R}(\alpha), \mathfrak{R}(\beta))>0 ; a, b, c, d, e, f, g, h, k \in \mathbb{C}, \mathfrak{R}(p) \geq 0)$.

Theorem 1. Let $x>0, \mathfrak{R}(p) \geq 0$, and the parameters $\mu, \mu^{\prime}, \nu, v^{\prime}, \eta, \rho, \gamma \in \mathbb{C}$ be such that

$$
\begin{aligned}
& \operatorname{Re}(\eta)>0, \\
& \mathfrak{R}(\rho)>\max \left\{0, \mathfrak{R}\left(\mu+\mu^{\prime}+\nu-\eta\right), \mathfrak{R}\left(\mu^{\prime}-\nu^{\prime}\right)\right\} .
\end{aligned}
$$

Then there holds the following fractional integral formula:

$$
\begin{aligned}
& \left(I_{0, x}^{\left(\mu, \mu^{\prime}, v, \nu^{\prime}, \eta\right)}\left[t^{\rho-1} F_{p}^{(\alpha, \beta)}(a, b ; c ; \gamma t)\right]\right)(x) \\
& =x^{\rho+\eta-\mu-\mu^{\prime}-1} \\
& \times \frac{\Gamma(\rho) \Gamma\left(\rho+\nu^{\prime}-\mu^{\prime}\right) \Gamma\left(\rho+\eta-\mu-\nu-\mu^{\prime}\right)}{\Gamma\left(\rho+\nu^{\prime}\right) \Gamma\left(\rho+\eta-\mu-\mu^{\prime}\right) \Gamma\left(\rho+\eta-v-\mu^{\prime}\right)} \\
& { }_{3} F_{p, 3}^{(\alpha, \beta)}\left[\begin{array}{cc}
a, b, \rho, \rho+\nu^{\prime}-\mu^{\prime}, \rho+\eta-\mu-\nu-\mu^{\prime} ; & \gamma x \\
c, \rho+\nu^{\prime}, \rho+\eta-\mu-\mu^{\prime}, \rho+\eta-v-\mu^{\prime} ; &
\end{array}\right] .
\end{aligned}
$$

Proof. For convenience, we denote the left-hand side of the result (12) by $\Delta(x)$. Then, applying Definition (1) and changing the order of integration and summation, which is valid under the condition given with Theorem 1 , we get

$$
\begin{aligned}
& \Delta(x):=\left(I_{0, x}^{\left(\mu, \mu^{\prime}, \nu, \nu^{\prime}, \eta\right)}\left[t^{\rho-1} F_{p}^{(\alpha, \beta)}(a, b ; c ; \gamma t)\right]\right)(x) \\
&=\left(I _ { 0 , x } ^ { ( \mu , \mu ^ { \prime } , \nu , \nu ^ { \prime } , \eta ) } \left[t^{\rho-1} \sum_{n=0}^{\infty} a_{n} \frac{B_{p}^{(\alpha, \beta)}(b+n, c-b)}{B(b, c-b)}\right.\right. \\
&\left.\left.\times \frac{(\gamma t)^{n}}{n !}\right]\right)(x) \\
&=\sum_{n=0}^{\infty} \frac{(a)_{n} \frac{B_{p}^{(\alpha, \beta)}(b+n, c-b)}{B(b, c-b)}}{\cdot \frac{\gamma^{n}}{n !}\left(I_{0, x}^{\left(\mu, \mu^{\prime}, \nu, \nu^{\prime}, \eta\right)}\left[t^{\rho+n-1}\right]\right)(x) .}
\end{aligned}
$$

Now, we can make use of (8) with $\rho$ replaced by $\rho+n\left(n \in \mathbb{N}_{0}\right)$ and we thus get from (13)

$$
\begin{aligned}
\Delta(x):= & x^{\rho+\eta-\mu-\mu^{\prime}-1} \sum_{n=0}^{\infty}(a)_{n} \frac{B_{p}^{(\alpha, \beta)}(b+n, c-b)}{B(b, c-b)} \\
& \cdot \frac{\Gamma(\rho-\eta) \Gamma\left(\rho+\nu^{\prime}-\mu^{\prime}+n\right) \Gamma\left(\rho+\eta-\mu-\nu-\mu^{\prime}+n\right)}{\Gamma\left(\rho+\nu^{\prime}+n\right) \Gamma\left(\rho+\eta-\mu-\mu^{\prime}+n\right) \Gamma\left(\rho+\eta-\nu-\mu^{\prime}+n\right)} \\
& \times \frac{(\gamma x)^{n}}{n !} \\
= & x^{\rho+\eta-\mu-\mu^{\prime}-1} \\
& \times \frac{\Gamma(\rho) \Gamma\left(\rho+\nu^{\prime}-\mu^{\prime}\right) \Gamma\left(\rho+\eta-\mu-\nu-\mu^{\prime}\right)}{\Gamma\left(\rho+v^{\prime}\right) \Gamma\left(\rho+\eta-\mu-\mu^{\prime}\right) \Gamma\left(\rho+\eta-\nu-\mu^{\prime}\right)} \\
& \times \sum_{n=0}^{\infty}(a)_{n} \frac{B_{p}^{(\alpha, \beta)}(b+n, c-b)}{B(b, c-b)} \\
& \cdot \frac{(\rho)_{n}\left(\rho+\nu^{\prime}-\mu^{\prime}\right)_{n}\left(\rho+\eta-\mu-\nu-\mu^{\prime}\right)_{n}}{\left(\rho+v^{\prime}\right)_{n}\left(\rho+\eta-\mu-\mu^{\prime}\right)_{n}\left(\rho+\eta-v-\mu^{\prime}\right)_{n}} \\
& \times \frac{(\gamma x)^{n}}{n !} .
\end{aligned}
$$

Finally by interpreting the last member of (14) by means of the definition of ${ }_{3} F_{p, 3}^{(\alpha, \beta)}(\cdot)$, we obtain the right-hand side of (12). This completes the proof of Theorem 1.

Theorem 2. Let $x>0, \mathfrak{R}(p) \geq 0$, and the parameters $\mu, \mu^{\prime}, \nu, \nu^{\prime}, \eta, \rho, \gamma \in \mathbb{C}$ and satisfy the following inequalities:

$$
\begin{aligned}
& \mathfrak{R}(\eta)>0, \\
& 0<\mathfrak{R}(\rho)<1+\min \left\{\mathfrak{R}(-\nu), \mathfrak{R}\left(\mu+\mu^{\prime}-\eta\right),\right. \\
& \left.\mathfrak{R}\left(\mu+\nu^{\prime}-\eta\right)\right\} .
\end{aligned}
$$

Then the following generalized fractional integral formula holds true:

$$
\begin{aligned}
& \left(I_{x, \infty}^{\mu, \mu^{\prime}, v, \nu^{\prime}, \eta}\left[t^{\rho-1} F_{p}^{(\alpha, \beta)}\left(a, b ; c ;\left(\frac{\gamma}{t}\right)\right)\right]\right)(x) \\
& =x^{\rho+\eta-\mu-\mu^{\prime}-1} \\
& \quad \times \frac{\Gamma(1-\rho-\nu) \Gamma\left(1-\rho-\eta+\mu+\mu^{\prime}\right) \Gamma\left(1-\rho-\eta+\mu+\nu^{\prime}\right)}{\Gamma(1-\rho) \Gamma(1-\rho+\mu-\nu) \Gamma\left(1-\rho-\eta+\mu+\mu^{\prime}+\nu^{\prime}\right)} \\
& { }_{3} F_{p, 3}^{(\alpha, \beta)}\left[\begin{array}{c}
a, b, 1-\rho-\nu, 1-\rho-\eta+\mu+\mu^{\prime}, 1-\rho-\eta+\mu+\nu^{\prime} ; \\
c, 1-\rho, 1-\rho+\mu-\nu, 1-\rho-\eta+\mu+\mu^{\prime}+\nu^{\prime} ;
\end{array}\right] .
\end{aligned}
$$

Proof. For convenience, we denote the left-hand side of the result (16) by $\Omega(x)$. Then, by making use of Definition (1) and 
changing the order of integration and summation, which is justified under the conditions stated with Theorem 2, we get

$$
\begin{aligned}
& \Omega(x):=\left(I_{x, \infty}^{\left(\mu, \mu^{\prime}, \nu, \nu^{\prime}, \eta\right)}\left[t^{\rho-1} F_{p}^{(\alpha, \beta)}\left(a, b ; c ;\left(\frac{\gamma}{t}\right)\right)\right]\right)(x) \\
& =\left(I _ { x , \infty } ^ { ( \mu , \mu ^ { \prime } , v , \nu ^ { \prime } , \eta ) } \left[t^{\rho-1} \sum_{n=0}^{\infty} a_{n} \frac{B_{p}^{(\alpha, \beta)}(b+n, c-b)}{B(b, c-b)}\right.\right. \\
& \left.\left.\times \frac{(\gamma / t)^{n}}{n !}\right]\right)(x) \\
& =\sum_{n=0}^{\infty}(a)_{n} \frac{B_{p}^{(\alpha, \beta)}(b+n, c-b)}{B(b, c-b)} \\
& \cdot \frac{\gamma^{n}}{n !}\left(I_{x, \infty}^{\left(\mu, \mu^{\prime}, \nu, \nu^{\prime}, \eta\right)}\left[t^{\rho-n-1}\right]\right)(x) .
\end{aligned}
$$

Now, we can make use of (9) with $\rho$ replaced by $\rho-n\left(n \in \mathbb{N}_{0}\right)$ and we thus get from (17)

$$
\begin{aligned}
& \Omega(x)=x^{\rho+\eta-\mu-\mu^{\prime}-1} \sum_{n=0}^{\infty}(a)_{n} \frac{B_{p}^{(\alpha, \beta)}(b+n, c-b)}{B(b, c-b)} \\
& \cdot\left(\left(\Gamma(1-\rho-\nu+n) \Gamma\left(1-\rho-\eta+\mu+\mu^{\prime}+n\right)\right.\right. \\
& \left.\times \Gamma\left(1-\rho-\eta+\mu+\nu^{\prime}+n\right)\right) \\
& \times(\Gamma(1-\rho+n) \Gamma(1-\rho+\mu-\nu+n) \\
& \left.\left.\times \Gamma\left(1-\rho-\eta+\mu+\mu^{\prime}+\nu^{\prime}+n\right)\right)^{-1}\right) \cdot \frac{(\gamma / x)^{n}}{n !} \\
& =x^{\rho+\eta-\mu-\mu^{\prime}-1} \\
& \times \frac{\Gamma(1-\rho-\nu) \Gamma\left(1-\rho-\eta+\mu+\mu^{\prime}\right) \Gamma\left(1-\rho-\eta+\mu+\nu^{\prime}\right)}{\Gamma(1-\rho) \Gamma(1-\rho+\mu-\nu) \Gamma\left(1-\rho-\eta+\mu+\mu^{\prime}+\nu^{\prime}\right)} \\
& \times \sum_{n=0}^{\infty}(a)_{n} \frac{B_{p}^{(\alpha, \beta)}(b+n, c-b)}{B(b, c-b)} \\
& \cdot \frac{(1-\rho-\nu)_{n}\left(1-\rho-\eta+\mu+\mu^{\prime}\right)_{n}\left(1-\rho-\eta+\mu+\nu^{\prime}\right)_{n}}{(1-\rho)_{n}(1-\rho+\mu-\nu)_{n}\left(1-\rho-\eta+\mu+\mu^{\prime}+\nu^{\prime}\right)_{n}} \\
& \frac{(\gamma / x)^{n}}{n !} \text {. }
\end{aligned}
$$

Finally by interpreting the last member of (18) by means of the definition of ${ }_{3} F_{p, 3}^{(\alpha, \beta)}(\cdot)$, we obtain the right-hand side of (16). This completes the proof of Theorem 2.

Remark 3. It may be noted that, for $\mu^{\prime}=0$, Theorems 1 and 2 are immediately reduced to the known results due to Agarwal [6].

\section{Special Cases and Concluding Observations}

In this section, we consider some consequences of the main results derived in the preceding sections. If we set $\alpha=\beta$ in (12) and (16), respectively, then by the known formula due to Chaudhry et al. (see, e.g., [2]), Theorems 1 and 2 yield the following corollaries.

Corollary 4. Let $x>0, \mathfrak{R}(p) \geq 0$, and the parameters $\mu, \mu^{\prime}, \nu, \nu^{\prime}, \eta, \rho, \gamma \in \mathbb{C}$ be such that

$$
\begin{gathered}
\mathfrak{R}(\eta)>0, \\
\mathfrak{R}(\rho)>\max \left\{0, \mathfrak{R}\left(\mu+\mu^{\prime}+\nu-\eta\right),\right. \\
\left.\mathfrak{R}\left(\mu^{\prime}-\nu^{\prime}\right)\right\} .
\end{gathered}
$$

Then there holds the following fractional integral formula:

$$
\begin{aligned}
& \left(I_{0, x}^{\left(\mu, \mu^{\prime}, v, \nu^{\prime}, \eta\right)}\left[t^{\rho-1} F_{p}(a, b ; c ; \gamma t)\right]\right)(x) \\
& =x^{\rho+\eta-\mu-\mu^{\prime}-1} \\
& \quad \times \frac{\Gamma(\rho) \Gamma\left(\rho+v^{\prime}-\mu^{\prime}\right) \Gamma\left(\rho+\eta-\mu-v-\mu^{\prime}\right)}{\Gamma\left(\rho+v^{\prime}\right) \Gamma\left(\rho+\eta-\mu-\mu^{\prime}\right) \Gamma\left(\rho+\eta-v-\mu^{\prime}\right)} \\
& { }_{3} F_{p, 3}\left[\begin{array}{c}
a, b, \rho, \rho+\nu^{\prime}-\mu^{\prime}, \rho+\eta-\mu-v-\mu^{\prime} ; \\
c, \rho+v^{\prime}, \rho+\eta-\mu-\mu^{\prime}, \rho+\eta-v-\mu^{\prime} ;
\end{array}\right] .
\end{aligned}
$$

Corollary 5. Let $x>0, \mathfrak{R}(p) \geq 0$, and the parameters $\mu, \mu^{\prime}, \nu, \nu^{\prime}, \eta, \rho, \gamma \in \mathbb{C}$ and satisfy the following inequalities:

$$
\begin{gathered}
\mathfrak{R}(\eta)>0, \\
\mathfrak{R}(\rho)<1+\min \left\{\mathfrak{R}(-\nu), \mathfrak{R}\left(\mu+\mu^{\prime}-\eta\right),\right. \\
\left.\mathfrak{R}\left(\mu+\nu^{\prime}-\eta\right)\right\} .
\end{gathered}
$$

Then the following generalized fractional integral formula holds true:

$$
\begin{aligned}
& \left(I_{x, \infty}^{\mu, \mu^{\prime}, v, \nu^{\prime}, \eta}\left[t^{\rho-1} F_{p}\left(a, b ; c ;\left(\frac{\gamma}{t}\right)\right)\right]\right)(x) \\
& =x^{\rho+\eta-\mu-\mu^{\prime}-1} \\
& \times \frac{\Gamma(1-\rho-v) \Gamma\left(1-\rho-\eta+\mu+\mu^{\prime}\right) \Gamma\left(1-\rho-\eta+\mu+\nu^{\prime}\right)}{\Gamma(1-\rho) \Gamma(1-\rho+\mu-\nu) \Gamma\left(1-\rho-\eta+\mu+\mu^{\prime}+\nu^{\prime}\right)} \\
& { }_{3} F_{p, 3}\left[\begin{array}{cc}
a, b, 1-\rho-\nu, 1-\rho-\eta+\mu+\mu^{\prime}, 1-\rho-\eta+\mu+\nu^{\prime} & ; \\
c, 1-\rho, 1-\rho+\mu-\nu, 1-\rho-\eta+\mu+\mu^{\prime}+\nu^{\prime} ; & \frac{\gamma}{x}
\end{array}\right] .
\end{aligned}
$$

Remark 6. For $\mu^{\prime}=0$, Corollaries 4 and 5 are immediately reduced to the known results due to Agarwal [6].

Furthermore, if we set $p=0$ and then make use of the result (1), Theorems 1 and 2 yield the various fractional integral formulas for the generalized hypergeometric function ${ }_{2} F_{1}$. 
Next, it can be easily seen that the Saigo fractional integral operators, the Erdélyi-Kober fractional integral operators, the Riemann-Liouville fractional integral operator, and the Weyl fractional integral operator are special cases of the operators (6). Therefore, we can easily derive several further consequences of the main results derived in the preceding sections by setting $\mu^{\prime}=0, v=-\mu$, and $\nu=0$ in the operators (6) (see, e.g., [18]).

Therefore, we conclude this paper with the remark that the results obtained in this paper are useful in deriving certain composition formulas involving various fractional integral operators and generalized Gauss hypergeometric functions.

\section{Conflict of Interests}

The authors declare that they have no conflict of interests in this paper.

\section{Acknowledgments}

The authors take this opportunity to express their deepest thanks to the referees for their valuable comments and essential suggestions to improve this paper as in the present form.

\section{References}

[1] P. Agarwal, M. Chand, and S. D. Purohit, "A note on generating functions involving generalized Gauss hypergeometric functions," National Academy Science Letters. In press.

[2] M. A. Chaudhry, A. Qadir, H. M. Srivastava, and R. B. Paris, "Extended hypergeometric and confluent hypergeometric functions," Applied Mathematics and Computation, vol. 159, no. 2, pp. 589-602, 2004.

[3] E. Özergin, Some properties of hypergeometric functions, [Ph.D. thesis], Eastern Mediterranean University, North Cyprus, 2011.

[4] E. Özergin, M. A. Özarslan, and A. Altın, "Extension of gamma, beta and hypergeometric functions," Journal of Computational and Applied Mathematics, vol. 235, no. 16, pp. 4601-4610, 2011.

[5] H. M. Srivastava and J. Choi, Zeta and q-Zeta Functions and Associated Series and Integrals, Elsevier Science, London, UK, 2012.

[6] P. Agarwal, "Certain properties of the generalized Gauss hypergeometric functions," Applied Mathematics \& Information Sciences, vol. 8, no. 5, pp. 2315-2320, 2014.

[7] D. Baleanu, K. Diethelm, E. Scalas, and J. J. Trujillo, Fractional Calculus: Models and Numerical Methods, vol. 3 of Complexity, Nonlinearity and Chaos, World Scientific, 2012.

[8] D. Băleanu, O. G. Mustafa, and R. P. Agarwal, "On the solution set for a class of sequential fractional differential equations," Journal of Physics A: Mathematical and Theoretical, vol. 43, no. 38, article 385209, 2010.

[9] A. A. Kilbas, H. M. Srivastava, and J. J. Trujillo, Theory and Applications of Fractional Differential Equations, vol. 204 of North-Holland Mathematics Studies, Elsevier (North-Holland) Science Publishers, Amsterdam, The Netherlands, 2006.

[10] V. Kiryakova, “On two Saigo's fractional integral operators in the class of univalent functions," Fractional Calculus \& Applied Analysis, vol. 9, no. 2, pp. 159-176, 2006.
[11] V. Kiryakova, "A brief story about the operators of the generalized fractional calculus," Fractional Calculus \& Applied Analysis, vol. 11, no. 2, pp. 203-220, 2008.

[12] O. I. Marichev, "Volterra equation of Mellin convolution type with a Horn function in the kernel," Izvestiya Akademii Nauk BSSR. Seriya Fiziko-Matematicheskikh Nauk, no. 1, pp. 128-129, 1974 (Russian).

[13] A. C. McBride, Fractional Calculus and Integral Transforms of Generalized Functions, vol. 31 of Research Notes in Mathematics, Pitman, London, UK, 1979.

[14] M. Saigo, “On generalized fractional calculus operators," in Recent Advances in Applied Mathematics, pp. 441-450, Kuwait University, Kuwait, 1996.

[15] M. Saigo and N. Maeda, "More generalization of fractional calculus," in Transform Methods \& Special Functions, pp. 386400, IMI-BAS, Sofia, Bulgaria, 1998.

[16] H. M. Srivastava and P. Agarwal, "Certain fractional integral operators and the generalized incomplete hypergeometric functions," Applied Mathematics and Computation, vol. 8, no. 2, pp. 333-345, 2013.

[17] F. W. J. Olver, D. W. Lozier, R. F. Boisvert, and C. W. Clark, Eds., NIST Handbook of Mathematical Functions, National Institute of Standards and Technology, Cambridge University Press, Gaithersburg, Maryland, 2010.

[18] A. M. Mathai, R. K. Saxena, and H. J. Haubold, The H-Function : Theory and Applications, Springer, London, UK, 2010. 


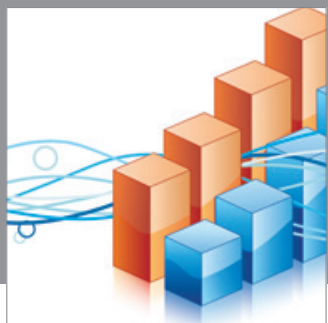

Advances in

Operations Research

mansans

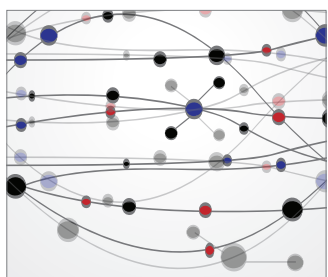

The Scientific World Journal
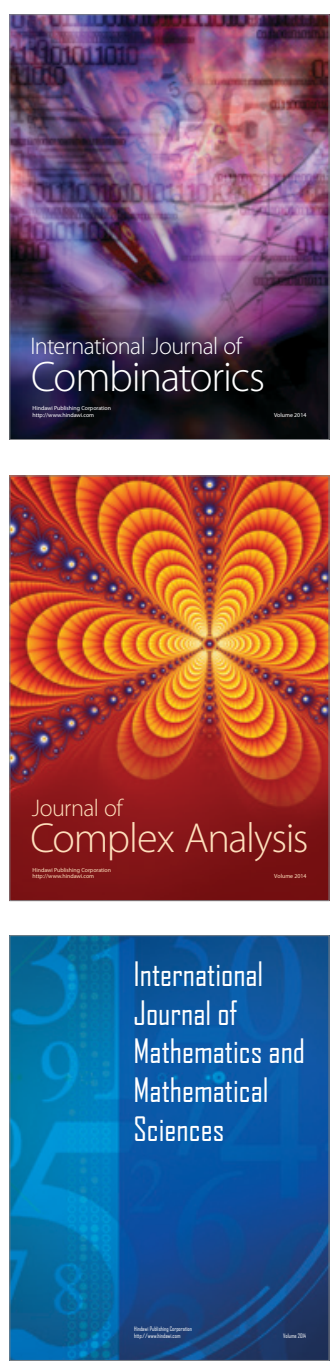
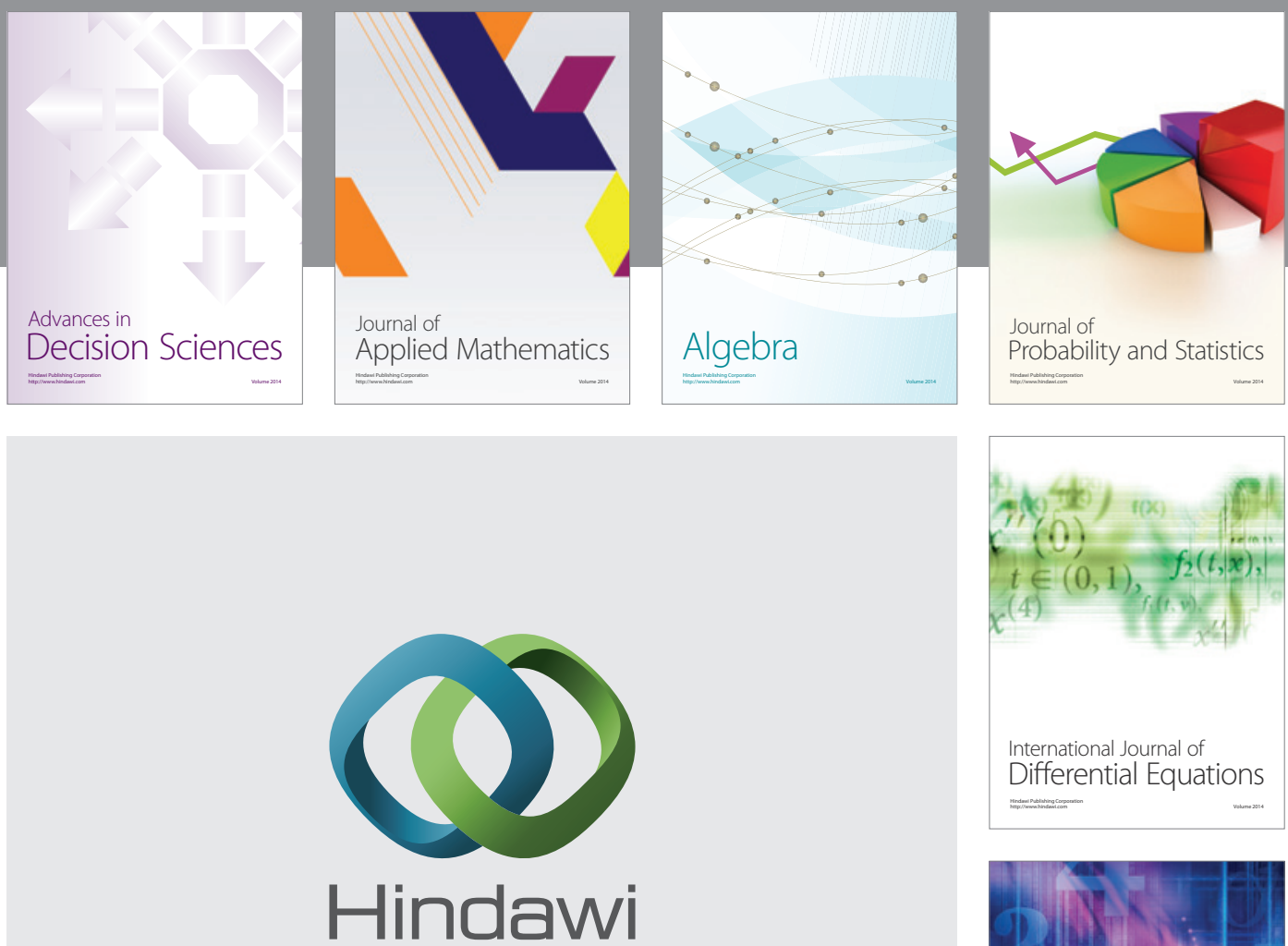

Submit your manuscripts at http://www.hindawi.com
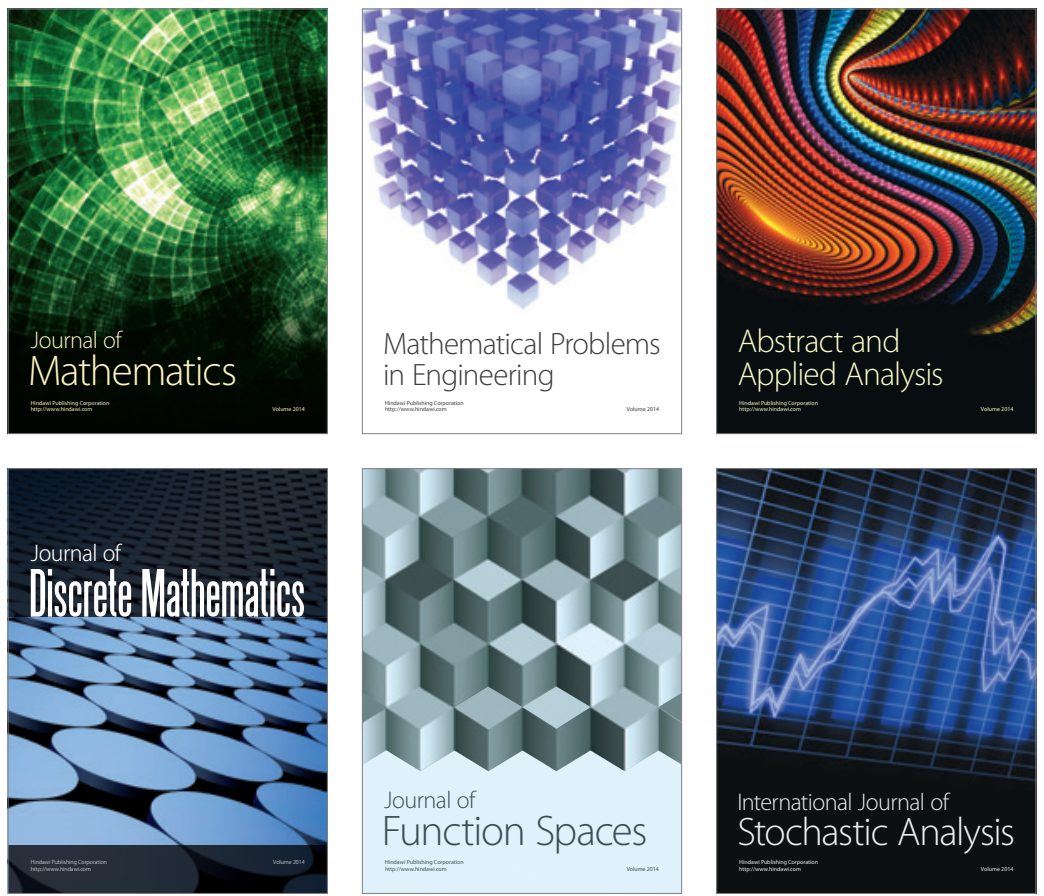

Journal of

Function Spaces

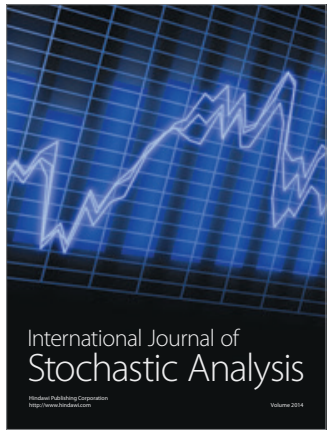

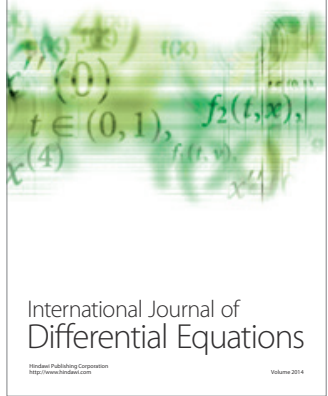
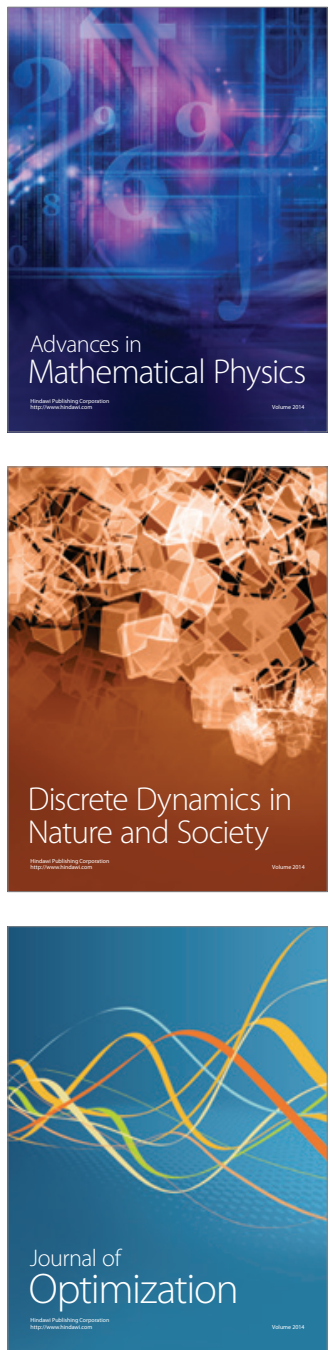